\title{
La economía creativa en Ecuador: un análisis retrospectivo en la provincia de Cotopaxi durante el período 2012 - 2015
}

\section{The creative economy in Ecuador: a retrospective analysis in the province of Cotopaxi during the period $2012-2015$}

\author{
Cristian Rodrigo Molina-Quinteros - ORCID ID 0000-0003-4191-7504 \\ María Elena Jerez-Calero - ORCID ID 0000-0002-0539-8746 \\ Luis Benigno Palomino-Siza - ORCID ID 0000-0001-8126-5083 \\ Universidad de las Fuerzas Armadas - ESPE, Ecuador
}

Autor para correspondencia: crmolina4@espe.edu.ec, mejerez@espe.edu.ec, lbpalomino@espe.edu.ec

Fecha de recepción: 17 de julio de 2018 Fecha de aceptación: 15 noviembre del 2018

Resumen: La economía creativa está orientada a servir de constructo integrador entre la creatividad, la innovación, el emprendimiento y la cultura; siendo el conjunto de actividades interrelacionadas que permiten el encadenamiento de las ideas, con el propósito de transformarlas en bienes y servicios culturales, teniendo en cuenta que cuyo valor estaría determinado por su contenido de propiedad intelectual. Tomando en consideración los enfoques teóricos existentes en el marco de esta teoría se la puede ubicar en el contexto de la corriente epistemológica del estructuralismo. A la vez, éste concepto en evolución está basado en el potencial de los activos creativos como generador de desarrollo y crecimiento económico, destacando que la creatividad es entendida como la capacidad de aportar nuevas respuestas e innovadoras prácticas empresariales frente a los retos que actualmente se enfrentan los individuos, las sociedades, las empresas y los territorios. Es así que, el objetivo de esta investigación apunta a divulgar la presencia que tiene este sector de la economía en la provincia de Cotopaxi - Ecuador; abordando el tema desde una perspectiva documental, la cual describe la radiografía retrospectiva del tejido empresarial perteneciente a la economía creativa en el periodo 2012 - 2015, basada en los datos del Directorio de Empresa del Instituto Nacional de Estadística y Censos.

Palabras clave: sectores creativos; empresas creativas; desarrollo urbano y territorial Abstract: The creative economy is oriented to serve as an integrating construct between creativity, innovation, entrepreneurship and culture; being the set of interrelated activities that allow the chaining of ideas, with the purpose of transforming them into goods and cultural services, taking into account that their value would be determined by their content of intellectual property. Taking into consideration the existing theoretical approaches within the framework of this theory, it can be located in the context of the epistemological current of structuralism. At the same time, this evolving concept is based on the potential of creative assets as a generator of development and economic growth, highlighting that creativity is understood as the ability to provide new answers and innovative business practices in the face of the challenges that currently are facing individuals, societies, companies and territories. Thus, the objective of this research aims to disseminate the presence of this sector of the economy in the province of Cotopaxi - Ecuador; approaching the subject from a documentary perspective, which describes the retrospective radiography of the 
business structure belonging to the creative economy in the period 2012 - 2015, based on the data of the Business Directory of the National Institute of Statistics and Census.

Key words: creative industries; creative enterprises; urban and territorial development

\section{Introducción}

La economía creativa en la última década ha ganado espacio en el tejido empresarial de las grandes ciudades del mundo; sin embargo, se ha visto un incremento en la brecha existencial entre el área urbana y el territorio de las pequeñas ciudades. Es así, como teóricos y académicos que abordan esta temática aparecen con nuevos postulados y propuestas para definir con más detalle los elementos que influyen en la composición de las empresas creativas. Partiendo de lo que mencionan Méndez, Michelini, Prada, y Tébar (2012), la creatividad es entendida como la capacidad de aportar respuestas nuevas y más eficaces prácticas empresariales frente a los retos a que se enfrentan individuos, sociedades y territorios.

Por otra parte, en el proceso de desarrollo de la calidad de vida, la ciudadanía se interesa por la innovación, indispensable para la economía creativa que tiene que asumir nuevos retos y cambios que impone el mercado global, que por cierto son altamente exigentes, y que lleva a los empresarios a considerar los conocimientos de la cultura y el arte para realizar un trabajo articulado y sistemático con todos los agentes que actúan en su cadena de valor (Martí, 2013), que desde la concepción de Porter (1987), es una sucesión de actividades que va añadiendo valor al producto o servicio que la compañía va generando para que el cliente le compre.

El interés que suscita la referencia a las industrias creativas, la clase creativa o ciudades creativas ha multiplicado la bibliografía científica dedicada a estas teorías, pese a las frecuentes imprecisiones que acompañan la traslación del concepto al ámbito de los estudios sobre desarrollo territorial. La economía naranja se halla en una dinámica de cambios rápidos que van evolucionando y se adaptan rápidamente a la situación en la que se encuentra, generando así oportunidades inmediatas, que hacen de este fenómeno, económicamente relevante (Buitrago y Duque, 2013).

Por otro lado, la llamada economía creativa va en exponencial crecimiento en el mundo, tanto en la ciencia como en las empresas. Es así, como la Organización de las Naciones Unidas para la Educación la Ciencia y la Cultura (UNESCO, 2013), en el informe sobre la economía creativa hace mención a que éste "es uno de los sectores de más rápido crecimiento en la economía mundial, no sólo en términos de generación de ingresos, sino también en lo que respecta a la creación de empleos y aumento de los ingresos en exportaciones". Así también, las cifras de la Conferencia de las Naciones Unidas sobre Comercio y Desarrollo (UNCTAD) publicadas en mayo del 2013 y citado por la UNESCO (2013), menciona que la economía creativa se ha convertido en un motor del desarrollo, es así como en el 2011 el comercio mundial de bienes y servicios creativos alcanzó 624 mil millones de dólares americanos, más del doble que entre 2002 y 2011. Durante este período, la tasa promedio de crecimiento anual del sector fue de $8,8 \%$, en tanto que la exportación de bienes creativos fue incluso mayor en los países en desarrollo, con un promedio anual del $12 \%$ respecto al mismo periodo.

Sin embargo, los conceptos de economía creativa aún no son sólidos en Latinoamérica, tanto en literatura científica, como en datos cuantitativos y cualitativos, en virtud a ello, en el 
Ecuador existe una presencia desproporcional de este sector de la economía, en vista de que son las áreas urbanas las que más crecen, dejando de lado a las periféricas (Prada Trigo y Jiménez Idrovo, 2014).

En este orden de ideas, es necesario realizar un análisis crítico en torno a las teorías vinculantes al tema; para posterior a ello, continuar con la indagación retrospectiva de las empresas del sector creativo en Cotopaxi, a fin de contrastar los resultados y valorar la presencia de industrias pertenecientes a este sector económico.

Finalmente, es importante mencionar que las cifras analizadas en este artículo, están disponibles en la base de datos del directorio de empresas del Instituto Nacional de Estadística y Censo (INEC). El período analizado corresponde a los años 2012 al 2015; los cuales permiten identificar la evolución de los establecimientos comerciales de la provincia pertenecientes a éste sector de la economía. La Clasificación Industrial Internacional Uniforme (CIIU) se encuentra desagregada a cuatro dígitos para una mejor comprensión, además permite identificar claramente las actividades empresariales pertenecientes a la economía creativa.

\section{Principales argumentos de la economía creativa}

La creatividad es entendida como la herramienta para aportar valor simbólico y generar innovaciones eficientes y eficaces a la empresarialidad. Desde el año 2000, la economía creativa ha revolucionado el mundo empresarial, económico y social; basado en una de las primeras teorías de Howkins (2001), quién define a la economía creativa como uno de los sectores más importantes en el desarrollo individual y colectivo, ya que esta actividad tiene diferentes valores y modelos de negocio, que están unidos por personas apasionados de la creación, la innovación y el desarrollo de nuevas ideas como parte de su trabajo diario. Estas personas generan un valor no tradicional, es decir, se esfuerzan en generar un valor más alto que los valores mercantilistas tradicionales, lo que significa, generar valor simbólico a los productos y servicios. Al tenor de lo expuesto, el especialista comenta que una economía creativa debe ser incluyente, ya que en ella están inmersos diferentes segmentos como: la propiedad intelectual, la propiedad industrial, las marcas, el diseño, el arte, la cultura, el entretenimiento, los medios de comunicación, la innovación y las denominadas industrias culturales y creativas.

No obstante, el término se ha desarrollado cada vez más, y actualmente, al hablar de economía creativa se habla desde la ciencia y tecnología, hasta la investigación y desarrollo (I+D). Por tanto, aun cuando se reconozcan las actividades y los procesos culturales como el núcleo de una nueva y poderosa economía, también se ocupa de manifestaciones creativas en ámbitos que no serían contemplados como "culturales" (PNUD, 2014).

El principio de toda diferenciación radica en la mente de las personas. El valor de lo simbólico expresado desde el producto o servicio, parte de la oferta y se canaliza en una percepción que tiene como destino "la mente del consumidor", esto se lo consigue con la generación de valor simbólico de los productos o servicios, generados por la lógica del consumo, lógica que está fundamentada en la esencia simbólica de la demanda y que se constituye en la lógica misma del marketing estratégico más allá de las diferencias propias de cada negocio (Wilensky, 1997). 
Por otro lado, para la UNCTAD, (2006) y citado por Casani, Rodríguez-Pomeda, y Sánchez (2011), la economía creativa, se encuentra en evolución y está basada en el potencial de los activos creativos para generar desarrollo y crecimiento económico. En el informe del año 2010 de Organización de las Naciones Unidas (ONU), hace mención a que la economía creativa ha crecido mucho más rápido que el resto de los sectores en la mayoría de los países desarrollados. Además, indica que son actividades intensivas en mano de obra cualificada, por lo que influyen sobre la generación de empleo de alta calidad y favorecen la inclusión de toda la sociedad y la diversidad cultural.

Bajo este contexto, cabe destacar que el especialista comenta que, dentro del contexto de economía creativa es necesario analizar el uso masivo y desarrollo de las redes sociales. Gracias al apoyo del internet han logrado dar un gran salto en la transformación de las actividades económicas generadas de forma centralizada y normalmente dependientes de las grandes empresas, pues el modelo de ruptura de la cadena de valor tradicional y aparición de negocios creativos, está enfocado en lograr la penetración desde el interior de los mercados, es decir, que los clientes tengan participación en las actividades empresariales, y para ellos el trabajo en redes es lo más indicado, ya que en estos espacios es en donde se genera un valor de una forma mucho más interactiva y descentralizada que la tradicional.

Por otro lado, el concepto de modelo de negocio en la economía creativa: emociones y redes sociales, se ha utilizado mucho en los últimos años para hacer referencia a la posibilidad de crear, producir y distribuir servicios o productos sin prácticamente poseer activos tangibles (Casani, Rodríguez-Pomeda, \& Sánchez, 2011). Todo ello ha cambiado la estructura de organización de las actividades económicas tradicionales, gracias a la correlación de las tecnologías de la información y las telecomunicaciones con las actividades multimedia, logrado así conducir a una importante transformación en la forma de producir, distribuir y consumir los contenidos creativos. Esta situación representa una amenaza para los grandes oligopolios que han dominado durante décadas estas industrias y, al mismo tiempo, una gran oportunidad para aquellas nuevas Pymes capaces de desarrollar modelos de negocio que posibiliten el aprovechamiento económico de las nacientes posibilidades tecnológicas y estratégicas, a través de la explotación de recursos y capacidades que permitan ofrecer productos diferenciados que generan valor simbólico en los usuarios.

Por lo tanto, la economía creativa se trata de un sector que reúne a las actividades que tienen como base para su producción la cultura y la creatividad, es decir, que los países abundantes en patrimonio cultural y producción intelectual, podrían encontrar en la economía creativa un motor de desarrollo (Deheinzelin, 2009).

\section{El modelo de la economía creativa denominado economía naranja}

Para Buitriago y Duque (2013), la economía naranja es un complejo cúmulo de términos, que no se necesita conceptualizarla definitivamente, ya que la economía naranja, se encuentra en una dinámica de cambios rápidos que va evolucionando y se adapta rápidamente a la situación en la que se encuentra, generando así oportunidades inmediatas, que hacen de él un fenómeno económicamente relevante. Sin embargo, propone una explicación de estilo creativo, 
argumentando que, la economía naranja es el conjunto de actividades interrelacionadas que permiten el encadenamiento de las ideas a fin de transformarlas en bienes y servicios culturales, cuyo valor está determinado por su contenido de propiedad intelectual.

El mundo de la economía naranja está conformado por dos sectores: el primero es la economía cultural y las industrias creativas, y en medio de estas, se ubica las industrias culturales convencionales; y el segundo son las áreas de soporte para la creatividad, en este aspecto cabe resaltar que la economía cultural incluye las actividades artísticas tradicionales. Las industrias creativas, son el conjunto de industrias culturales convencionales, las cuales forman parte del grupo de creaciones funcionales, nuevos medios y software, y actividades relativas a la construcción de un patrimonio cultural y su transmisión, como por ejemplo: artes visuales, escénicas y espectáculos. Mientras que, las áreas de soporte para la creatividad son: a) investigación, desarrollo, e innovación creativa y cultural, b) formación técnica especializada en actividades recreativas, c) gobernanza (institucionalidad) y derechos de propiedad intelectual y d) educación profesional creativa.

Entonces, se puede decir que la principal causa de la ruptura de la cadena de valor tradicional, es producida por la distribución o plaza del marketing mix, que tradicionalmente estaba controlada por las grandes empresas, quienes eran las que manejaban los embudos por los cuales tenían que pasar todas las posibles iniciativas o emprendimientos que quisieran alcanzar el mercado. Con el desarrollo del Internet, las Pymes pueden estar más cerca de los mercados y disponer de los recursos adecuados para aprovechar sus beneficios, a través de la utilización del nuevo entorno tecnológico. Las Pymes están en la posibilidad de saltarse los canales de distribución tradicionales, pudiendo llegar directamente al consumidor, generando así una ruptura en la agregación de valor tradicional, pues lo que anteriormente era una gran ventaja para las grandes empresas, hoy se convierte en su principal problema.

\section{Métodos}

\section{Fuente de información:}

Para realizar el estudio de los sectores de la economía creativa en la provincia de Cotopaxi, se recopiló información secundaria a través de la base de datos publicada por el INEC. Este organismo presenta de manera permanente, a través del Directorio de Empresas información actualizada sobre los establecimientos empresariales. Esta fuente se considera como adecuada, pues constituye un marco muestral relevante para el análisis e investigación. Es así que, el Sistema Integrado de Consultas administrado por el INEC permitió cruzar las variables y filtrar los datos a nivel de provincia.

\section{Nivel y tipo de investigación:}

La investigación aplicada fue descriptiva y longitudinal, se procesó información de cuatro años para analizar la evolución de las empresas pertenecientes a los sectores creativos. La información corresponde a datos históricos a partir del año 2012 al 2015. Las unidades de observación y análisis fueron las empresas y establecimientos de las industrias creativas constituidas legalmente en la provincia de Cotopaxi. Las empresas se concentraron en nueve 
grupos que conforman el sector de la economía creativa. Fue necesario considerar la Clasificación Industrial Internacional Uniforme (CIIU) a cuatro dígitos para segmentar el universo de las empresas.

\section{Selección de la población:}

La economía creativa está formada por nueve grupos, los cuales fueron determinados de acuerdo al código de Clasificación Industrial Internacional Uniforme (CIIU), a saber: artes visuales ; sitios culturales ; expresiones culturales y tradicionales; artes escénicas; audiovisuales; media interactiva; servicios creativos; diseño, y finalmente el grupo de literatura y publicaciones; es importante señalar que las empresas de este sector fueron agrupadas considerando el componente de creatividad, innovación y dinamismo.

Con la información recopilada se realizó un análisis estadístico de la evolución anual y del cuatrienio evaluado. Además, se determinó la composición del sector a lo largo del tiempo, y los resultados obtenidos permitieron comprender la dinámica de la economía creativa en la provincia de Cotopaxi.

\section{Resultados}

La recolección de información se realizó a partir de la base de datos del Directorio de Empresas publicada por el Instituto Ecuatoriano de Estadísticas y Censos (INEC).

Tabla 1.Composición de las empresas del sector creativo

\begin{tabular}{llllll}
\hline No. & GRUPO & $\mathbf{2 0 1 2}$ & $\mathbf{2 0 1 3}$ & $\mathbf{2 0 1 4}$ & $\mathbf{2 0 1 5}$ \\
\hline $\mathbf{1}$ & Artes visuales & $1,4 \%$ & $1,3 \%$ & $1,3 \%$ & $1,5 \%$ \\
$\mathbf{2}$ & Sitios culturales & - & - & - & $0,2 \%$ \\
$\mathbf{3}$ & Expresiones culturales y tradicionales & $8,8 \%$ & $8,6 \%$ & $7,6 \%$ & $9,9 \%$ \\
$\mathbf{4}$ & Artes escénicas & $3,9 \%$ & $3,9 \%$ & $3,7 \%$ & $5,5 \%$ \\
$\mathbf{5}$ & Audiovisuales & $19,4 \%$ & $18,5 \%$ & $18,7 \%$ & $4,7 \%$ \\
$\mathbf{6}$ & Media interactiva & $0,3 \%$ & $0,3 \%$ & $0,4 \%$ & $1,3 \%$ \\
$\mathbf{7}$ & Servicios creativos & $24,8 \%$ & $25,5 \%$ & $26,7 \%$ & $59,3 \%$ \\
$\mathbf{8}$ & Diseño & $41,4 \%$ & $41,9 \%$ & $41,7 \%$ & $17,6 \%$ \\
$\mathbf{9}$ & Literatura y publicaciones & - & - & - & - \\
Total & & $100 \%$ & $100 \%$ & $100,0 \%$ & $100 \%$ \\
Total empresas sectores creativos & 1.301 & 1.366 & 1.500 & 1.040 \\
Total empresas provincia & 31.895 & 34.959 & 31.895 & 40.133 \\
\hline
\end{tabular}

Nota. Datos obtenidos del INEC. Elaborado por: Los Autores.

De la Tabla 1 se desprende que, durante el año 2012, la participación de las empresas de la provincia de Cotopaxi que se encuentran ubicadas en el Sector Creativo es pequeña, apenas el $4.08 \%$ pertenecen a este sector en función del total existente a nivel provincial. En primer lugar en este sector se destacan las actividades relacionadas con el grupo de Diseño, las cuales representan el 41.4\%, siendo la fabricación de prendas de vestir las que mayor presencia tienen. En segundo lugar se ubica el grupo de Servicios Creativos con una participación de 24.8\%, evidenciándose que las actividades de arquitectura e ingeniería y actividades conexas de 
consultoría técnica son las más representativas. En tercer lugar se tiene al grupo de Audiovisuales con un 19.4\%, siendo otras actividades de telecomunicación las que lideran este grupo. La participación de estos tres grupos, durante los años 2013 y 2014 se mantiene sin mayores variaciones.

En el año 2015 este esquema cambia drásticamente, es así que el grupo de Servicios Creativos pasa a ocupar el primer lugar con un 59.3\%, donde la actividad que despunta en participación son las relacionadas a otras actividades profesionales, científicas y técnicas. El grupo Diseño pasa al segundo lugar con un $17.6 \%$ debido a que las actividades de fabricación de prendas de vestir se redujeron considerablemente al pasar de 447 establecimientos en el 2014 a 27 en 2015; y, en tercer lugar se ubica el grupo Expresiones culturales y tradicionales en el que se ubican otras actividades de esparcimiento y recreativas.

En la provincia de Cotopaxi, no existe registro de ningún establecimiento en el grupo de literatura y publicaciones, en este se ubican aquellas que se dedican a la publicación de bases de datos que están sujetas a protección en su forma, pero no en su contenido como por ejemplo: directorios telefónicos, compilaciones, compendios farmacéuticos, entre otros.

Llama la atención que en el grupo correspondiente a Sitios Culturales, no exista ningún establecimiento que se ubique en esta categoría, solo en el año 2015 se registra la existencia de 2 bibliotecas en la provincia.

Se puede evidenciar que durante el año 2015, las empresas pertenecientes al sector creativo cayó un 30.67\%; y tan solo representan un $2.59 \%$ del total de establecimientos de la provincia. Esta situación es contradictoria, puesto que el número de establecimientos en la provincia se incrementó en un $25.83 \%$.

A continuación, se presenta un análisis de la variación anual de las empresas del sector creativo.

Tabla 2

\begin{tabular}{lllll}
\hline No. & GRUPO & $\begin{array}{l}\text { Variación } \\
\mathbf{2 0 1 2} \mathbf{- 2 0 1 3}\end{array}$ & $\begin{array}{l}\text { Variación } \\
\mathbf{2 0 1 3 - 2 0 1 4}\end{array}$ & $\begin{array}{l}\text { Variación } \\
\mathbf{2 0 1 4} \mathbf{- 2 0 1 5}\end{array}$ \\
\hline $\mathbf{1}$ & Artes visuales & $0 \%$ & $5,6 \%$ & $-15,8 \%$ \\
$\mathbf{2}$ & Sitios culturales & - & - & - \\
$\mathbf{3}$ & Expresiones culturales y tradicionales & $3,5 \%$ & $-3,4 \%$ & $-9,6 \%$ \\
$\mathbf{4}$ & Artes escénicas & $3,9 \%$ & $5,7 \%$ & $1,8 \%$ \\
$\mathbf{5}$ & Audiovisuales & $0,0 \%$ & $10,7 \%$ & $-82,5 \%$ \\
$\mathbf{6}$ & Media interactiva & $0,0 \%$ & $50,0 \%$ & $116,7 \%$ \\
$\mathbf{7}$ & Servicios creativos & $7,7 \%$ & $14,9 \%$ & $54,3 \%$ \\
$\mathbf{8}$ & Diseño & $6,3 \%$ & $9,3 \%$ & $-70,7 \%$ \\
$\mathbf{9}$ & Literatura y publicaciones & - & & - \\
\hline
\end{tabular}

Nota. Datos obtenidos del INEC. Elaborado por: Los Autores.

Las empresas de la economía creativa en la provincia de Cotopaxi, no reportan una variación significativa en cuanto al número de establecimientos. A partir de la Tabla 2 se concluye que, durante el período 2012 - 2013, las empresas del grupo de Servicios creativos registran un crecimiento de $7.7 \%$, en el período 2013 - 2014 esta tendencia continúa, y el 
incremento es de $14.9 \%$; la variación más importante se da durante el último período, con un aumento de $54.3 \%$ de establecimientos en este grupo.

Por otro lado, las empresas que pertenecen al grupo de Media Interactiva, en el primer período se mantiene sin variaciones, para el segundo período tiene un incremento significativo del $50 \%$ y para el tercer período estas se duplican. Las actividades que tienen mayor crecimiento son: procesamiento de datos, hospedaje y actividades conexas, y, actividades de programación informática.

Los otros grupos, sufrieron un decrecimiento importante durante el 2014 - 2015. El grupo de Audiovisuales, tiene el resultado más desalentador, presenta una disminución de $82.5 \%$, siendo las empresas que se dedican a otras actividades de telecomunicaciones las más afectadas. El grupo Diseño también se contrae, el número de establecimientos se redujo en $70.7 \%$. Una contracción del $15.8 \%$ sufre las empresas del grupo Artes visuales, en la que existe un solo tipo de actividad, la fotografía.

El análisis de la variación de las empresas a lo largo del período en análisis, se presenta a continuación:

Tabla 3.

\begin{tabular}{lllllll}
\hline No. & GRUPO & $\mathbf{2 0 1 2}$ & $\mathbf{2 0 1 3}$ & $\mathbf{2 0 1 4}$ & $\mathbf{2 0 1 5}$ & $\begin{array}{l}\text { Variación } \\
\text { Del } \\
\text { Período }\end{array}$ \\
\hline $\mathbf{1}$ & Artes visuales & & & & & $-3,8 \%$ \\
$\mathbf{2}$ & Sitios culturales & 18 & 18 & 19 & 16 & - \\
$\mathbf{3}$ & Expresiones culturales y tradicionales & 114 & 118 & 114 & 103 & $-3,3 \%$ \\
$\mathbf{4}$ & Artes escénicas & 51 & 53 & 56 & 57 & $3,7 \%$ \\
$\mathbf{5}$ & Audiovisuales & 253 & 253 & 280 & 49 & $-41,8 \%$ \\
$\mathbf{6}$ & Media interactiva & 4 & 4 & 6 & 13 & $47,5 \%$ \\
$\mathbf{7}$ & Servicios creativos & 323 & 348 & 400 & 617 & $23,8 \%$ \\
$\mathbf{8}$ & Diseño & 538 & 572 & 625 & 183 & $-29,9 \%$ \\
$\mathbf{9}$ & Literatura y publicaciones & - & - & - & - & - \\
Total empresas sectores creativos & 1.301 & 1.366 & 1.500 & 1.040 & \\
Total empresas provincia & 31.895 & 34.959 & 31.895 & 40.133 & \\
\hline
\end{tabular}

Nota. Datos obtenidos del INEC. Elaborado por: Los Autores.

Durante el cuatrienio, son las empresas de Media Interactiva, Servicios Creativos y Artes escénicas las que evolucionan favorablemente, el crecimiento promedio es de $47.5 \%, 23.8 \%$ y $3,7 \%$ respectivamente. Son cuatro grupos de las empresas creativas que reducen su presencia en el mercado, a saber: Audio visuales 41.8\%, Diseño 29.9\%, Artes visuales 3.8\% y Expresiones culturales $3.3 \%$. Estos resultados denotan que, la economía creativa tiene escasa participación y tanto el sector público como el privado no han realizado los esfuerzos necesarios para dar impulso a este sector, por el contrario se evidencia que el número de establecimientos se redujo considerablemente.

\section{Discusión}


Los resultados obtenidos en la investigación establecen que el número de empresas pertenecientes a la economía creativa en la provincia de Cotopaxi es escasa. Éste sector económico representa el $4.08 \%$ en relación al total de empresas registradas en la base de datos del Directorio de Empresas del INEC a nivel provincial. Los resultados económicos de este sector en América Latina y El Caribe dan cuenta que se generaron 124.000 millones de dólares por concepto de ingresos y se crearon 1.9 millones de fuentes de empleo. Sin embargo en la provincia el panorama es poco alentador, pues la creación y permanencia en el mercado de este tipo de industrias es poco significativa.

Sin embargo, de ese $4.08 \%$ identificado como actividades que se desarrollan como economía naranja, el $41.4 \%$ pertenecen al grupo de Diseño, es decir, en la provincia se han registrado cerca de 447 establecimientos que están dedicados a la confección de prendas de vestir considerando los años del 2012 al 2014; sin embargo, esta participación se ve mermada durante el 2015, periodo en el cual el grupo de Diseño obtiene una representatividad del $17.6 \%$, disminuyendo 23.8 puntos porcentuales. Esta cifra inquieta e incide negativamente en el desempeño de la economía local, sumado a tres acotamientos de importancia como: la reactivación de la actividad del volcán Cotopaxi, la recesión económica del país y los bajos precios del petróleo, los cuales conjugaron un escenario poco favorable para este sector creativo.

Por otro lado, hay que mencionar que los grupos que particularmente llaman la atención son: Literatura y publicaciones y Sitios culturales; pues estos grupos tienen una presencia de cero puntos en los años evaluados; únicamente en el 2015 se registraron dos bibliotecas en toda la provincia de Cotopaxi. La data desactualizada fue el limitante al momento de desarrollar de la investigación, pues la información más reciente corresponde al año 2015 publicada en el Directorio de Empresas del INEC.

\section{Conclusiones}

Las empresas que pertenecen a los sectores de la economía creativa, tienen como principal característica el grado de innovación e incorporación de mano de obra calificada para la elaboración de los productos y/o servicios. Alrededor del mundo se evidencia que la economía creativa es un motor de desarrollo, por lo cual su crecimiento trae resultados positivos para las zonas en las que este concepto está dentro de sus agendas políticas.

En la provincia de Cotopaxi, de acuerdo al estudio realizado, este sector tiene en la actualidad una escasa participación, originado principalmente por la ausencia de políticas gubernamentales que promuevan la expansión de la I+D+i. Los grupos que tienen mayor importancia son: Servicios creativos y Diseño, los cuales concentran en promedio el $67 \%$ de las empresas que pertenecen a éste sector de la economía.

Por otro lado, la evolución de estas empresas durante el período de análisis, no es significativa, puesto que de manera global sufren un decrecimiento del 3.8\%, situación que es fruto del cierre de muchas empresas debido a que la economía de la provincia y del país tuvo que afrontar los efectos ocasionados por la reactivación del volcán Cotopaxi en el año 2015.

Para finalizar se puede indicar que, los países latinoamericanos están interesados en brindar el apoyo a estos sectores, es así que en Chile, Colombia, y México han aplicado políticas 
tendientes a impulsar el desarrollo creativo. En el Ecuador la promulgación del Código Orgánico de la Economía Social de los Conocimientos, Creatividad e Innovación es un primer paso hacia el desarrollo de la economía creativa.

\section{Bibliografía}

Bindi, R. D. (2006). Creación de Valor en los Agro-Negocios a partir del Producto Imaginario. CYTA, Buenos Aires.

Buitriago, F., \& Duque, I. (2013). La economía naranja. Una oportunidad infinita. Bogotá: BID.

Casani, F., Rodríguez-Pomeda, J., \& Sánchez, F. (2011). Los nuevos modelos de negocio en la economía creativa: emociones y redes sociales. Universia Business Review, pp. 50-57.

Deheinzelin, L. (2009). Proyecto de difusión y actuación en economía creativa y desarrollo sostenible. Sao Paulo, Brasil: Instituto Suba.

Guerras, L., \& Navas, J. (2015). La Dirección Estratégica de la Empresa. Navarra, España: Thomson.

Howkins. (2001). the creative economy: How people make money from ideas. Segunda Edición. London, Reino Unido, Penguin Books.

López, B. (2007). Publicidad Emocional. Estrategias creativas. Madrid, España: ESIC.

Martí, E. J. (2013). Clústers. Barcelona, España: Ediciones Urano.

Méndez, R., Michelini, J. J., \& Prada, J. (2012). Economía creativa y desarrollo urbano en España: una aproximación a sus lógicas espaciales. EURE, Santiago, pp.10-20.

Méndez, R., Michelini, J., Prada, J., \& Tébar, J. (2012). Economía creativa y desarrollo urbano en España: una aproximación a sus lógicas espaciales. EURE, pp. 5-32.

Organización de las Naciones Unidas para la Educación la Ciencia y la Cultura. (2013). Informe sobre la Economía Creativa. México: UNESCO, pp. 153.

Petracca, E. (2004). La Creación de Valor para el Accionista y el Consumidor. Erald Management.

PNUD, U. (2014). Informe sobre la Economía Creativa 2013. México DF, México.

Porter, M. (1987). From Competitive Advantage to Corporate Strategy. Nueva York: Harvard Business School.

Prada Trigo, J., \& Jiménez Idrovo, Í. (2014). La economía creativa en Ecuador: una aproximación a sus lógicas espaciales a partir del censo nacional económico de 2010. Entorno geográfico, pp.110-135.

Wilensky, A. (1997). Marketing Estratégico. Fondo de Cultura Económica, Buenos Aires, Argentina. 